\section{PWE-084 RELATIONSHIP BETWEEN INDICES OF FRAILTYI DISABILITY WITH DISEASE SEVERITY AND NUTRITIONAL STATUS IN PATIENTS WITH CIRRHOSIS}

${ }^{1}$ Priya Dhar*, ${ }^{2}$ Lynda Greenslade, ${ }^{2}$ Rachel Westbrooke, ${ }^{3}$ Clive D Jackson, ${ }^{2}$ Aileen Marshall, ${ }^{1}$ Marsha Yvonne Morgan*. ' UCL Institute for Liver and Digestive Health, University College London, London, UK; ${ }^{2}$ The Sheila Sherlock Liver Centre, Royal Free Hospital, London, UK;

${ }^{3}$ The Department of Neurophysiology, Royal Free Hospital, London, UK

\subsection{6/gutjnl-2018-BSGAbstracts.226}

Introduction Disease severity and nutritional status are important determinants of outcome in patients with cirrhosis. Health-related quality of life, mental health, disability and frailty all affect outcome. However it is unclear if these measures of functional decline provide information additional to that provided by conventional outcome measures. This study aimed to determine whether indices of functional decline correlate with nutritional status and disease severity.

Methods Eighty-six consecutive patients (mean [range] age 54.6 [24-81] yr: 59 men; mean MELD 12.7 [6-37]) attending the hepatology service at the Royal Free Hospital, London were included. All underwent the following assessment in a single sitting: disease severity: MELD; nutritional status: The Royal Free Hospital-Nutritional Prioritising Tool (RFH-NPT); quality of life: Chronic Liver Disease Questionnaire (CLDQ) and Euro Quol-5 Dimension Tool (EQ-5D); mental health: Beck Anxiety/Depression Indices (BAI; BDI); disability: Activities (ADL) and Independent Activities of Daily Living (IADL); frailty: Clinical Frailty Scale (CFS), Short Physical Performance Battery (SPPB) and Fried Frailty Criteria (FFC) plus two composite measures, the Bristol Prognostic Index and Karnofsky Age MELD Model (KAM).

Results There was a significant degree of cross correlation between the tools used to assess functional decline $(|\mathrm{R}|$ between 0.05-0.58). There were significant correlations between indices of functional decline and nutritional status with the exception of the disability indices (table 1). However, only the Bristol and KAM tools, which contain a measure of disease severity, correlated with MELD.

Conclusion In patients with cirrhosis indices of functional decline correlate with nutritional status but not with disease severity. These indices likely provide information of value in determining outcome not captured using conventional outcome measures. Information on their predicative validity would help determine their clinical utility.

\begin{tabular}{|c|c|c|}
\hline \multirow[t]{2}{*}{ Tool } & MELD & RFH-NPT \\
\hline & \multicolumn{2}{|l|}{$r$ ( $p$ value) } \\
\hline CLDQ & $0.04(0.74)$ & $-0.33(0.00)^{*}$ \\
\hline EQ-5D-5L & $-0.07(0.55)$ & $-0.36(0.00)^{*}$ \\
\hline BAI & $0.03(0.78)$ & $0.31(0.01)^{*}$ \\
\hline BDI & $0.13(0.26)$ & $0.38(0.00)^{*}$ \\
\hline ADL & $-0.14(0.22)$ & $-0.15(0.16)$ \\
\hline IADL & $-0.11(0.30)$ & $-0.19(0.08)$ \\
\hline CFS & $0.07(0.52)$ & $0.41(0.00)^{*}$ \\
\hline SPPB & $-0.2(0.09)$ & $-0.38(0.00)^{*}$ \\
\hline $\mathrm{FFC}$ & $0.04(0.7)$ & $0.47(0.00)^{*}$ \\
\hline Hand Grip & $0.01(0.96)$ & $-0.21(0.06)^{*}$ \\
\hline Bristol $^{a}$ & $0.28(0.01)^{*}$ & $0.32(0.00)^{*}$ \\
\hline $\mathrm{KAM}^{\mathrm{a}}$ & $0.34(0.00)^{*}$ & $0.37(0.00)^{*}$ \\
\hline
\end{tabular}

